\title{
Case report: Tuberculous mastoiditis an uncommon presentation of extrapulmonary tuberculosis in a young immunocompetant adult male
}

\author{
Pradeep Kumar Vyas ${ }^{1 *}$, Vidyadhara Lakkappan ${ }^{1}$, Suhas Thorat ${ }^{1}$ and Alan Andrade ${ }^{2}$ \\ ${ }^{1}$ Department of Critical Care Medicine, Holy Family Hospital, Bandra (West) Mumbai 400050, India \\ ${ }^{2}$ Department of E.N.T. St. Andrews Road Pali Hill, Bandra (West) Mumbai 400050, India
}

\begin{abstract}
Tuberculosis is a major public health problem in India, but tuberculous mastoiditis is quite rare, and is often diagnosed late. We report here the case of an 18-yearold male who had unusual chronic left mastoiditis and retro-auricular fistula, associated with spontaneous discharge of bony sequestrum. CT scan of temporal bones showed complete destruction of left mastoid cells. A left mastoidectomy confirmed the tuberculous mastoiditis diagnosis. The clinical outcome was favorable after anti-tuberculosis treatment. Otologic tuberculosis of the ear is well described in the literature and symptoms include chronic otorrhea with audio-vestibular symptoms. The specificity of this case is that the boy had an isolated involvement of the mastoid with spontaneous bony sequestrum discharge, but without otorrhea.
\end{abstract}

\section{Introduction}

In India tuberculosis is a major public health problem. It is a contagious disease transmission is usually due to droplet infection. According to $\mathrm{WHO}$, about 10 million people are infected in the world and 3 million die every year mostly in the developing countries $[1,2]$. It is caused by "mycobacterium tuberculosis" which can be very aggressive when there is local and/or general immune deficiency. The risk factors include very young or old age person, malnutrition and HIV infection [1,3]. Pulmonary system is mainly affected, followed by lymph nodes and visceral organs. Middle ear infections and tuberculous mastoiditis are rare manifestations. They represent less than $0.1 \%$ of all chronic suppurative otitis media [1,2]. The most common tuberculous mastoiditis may be secondary to spread of infection through the Eustachian tube, the haematogenous route or the tympanic membrane [1-4]. The diagnosis of tuberculous mastoiditis may be difficult due to variability of symptoms, which results in prolonged delays between the onset of symptoms and the diagnosis [1]. Here we present a case of tuberculous mastoiditis in an 18 years old male, initially difficult to diagnose with some potential complications.

\section{Background}

Tuberculous mastoiditis was first described by Jean Louis Petit in the 18th century; In1853 Wilde presented the classical picture of tuberculosis otitis media presented as painless ear discharge of insidious onset with multiple perforations in the tympanic membrane, and pale granulations in middle ear cleft. Politzer discussed the destructive nature of this disease, in 1882 Koch discovered tubercle bacilli [1]. The incidence of tuberculosis otitis media has been reported to be $0.04 \%$ to $0.9 \%$ of all Chronic Suppurative Otitis Media (CSOM) in the developed countries [2,3]. Tuberculosis affects the middle ear through three routes: aspiration of mucus through the Eustachian tube, haematogenous dissemination from other tuberculous foci or direct implantation through the external auditory canal and tympanic membrane perforation [1-3]. The incidence is more in the developing countries [4]. In recent years extra pulmonary tuberculosis has more frequently been associated with mastoiditis in patients with immunodeficiency state. Although tuberculosis of the mastoid or to mastoiditis is a very rare complication of tuberculosis today, when occurs it may cause significant morbidity, complications such as facial palsy and permanent hearing loss $[5,6]$. The diagnosis of tuberculous mastoiditis is difficult and is often delayed, especially if the patient presents with no other manifestations of the disease. Here we present a case of 18 years old man with tuberculous mastoiditis.

\section{Case report}

An 18 years old male h/o chronic OSM for 10 years, with no prior comorbidities presented with $\mathrm{h} / \mathrm{o}$ fever with chills headache, right ear pain and generalized weakness for 1 week, he also complaints of decreased appetite and loss of weight. On examination he was conscious oriented afebrile mildly dehydrated vitals were normal and hemodynamically stable. General and systemic examination were unremarkable. There was sensorineural hearing loss in right ear. His routine hemogram shows Hb 13.7 gm\%, WBC 5500/cu mm, platelets 230000. Dengue, malaria was negative. Liver and renal profile were normal. X-Ray chest normal. X-Ray right mastoid shows mastoiditis. Audiogram showed a right ear conductive loss $(35 \mathrm{~dB})$, with an air bone-gap of $25 \mathrm{~dB}$. CT scan of the temporal bones showed mastoiditis associated with irregular

${ }^{*}$ Correspondence to: Pradeep Kumar Vyas, Department of Critical Care Medicine, Holy Family Hospital St. Andrews Road, Bandra (West) Mumbai 400050, India, Tel: 91-9920696710 Email: drvyaspradeep@gmail.com

Key words: tuberculosis, tuberculous mastoiditis, bony sequestrum, fistula, mastoidectomy

Received: January 07, 2020; Accepted: January 17, 2020; Published: January 23 , 2020 

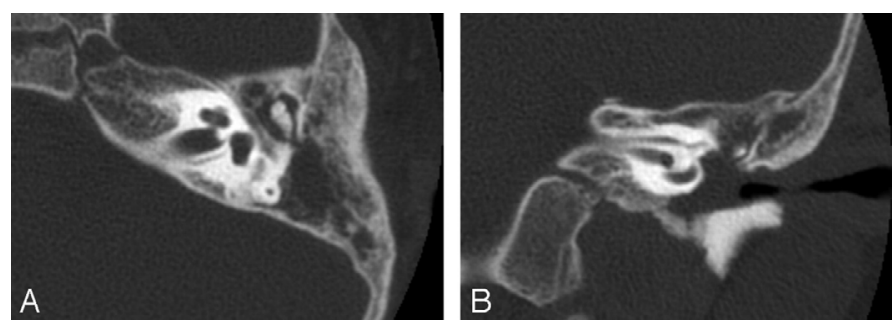

Figure 1A. Audiogram showed a right ear conductive loss ( $35 \mathrm{~dB})$, with an air bone-gap of $25 \mathrm{~dB}$. 1B. CT scan of the temporal bones showed mastoditis associated with irregular destruction in mastoid air cells and bony sequestrum, with small subdural abscess.

destruction in mastoid air cells and bony sequestrum, with small subdural abscess (Figures 1A and 1B). In regard to the atypical clinical presentation, a right mastoidectomy was therefore performed under general anaesthesia Granulomatous inflammation of mastoid mucosa was found with pus which was positive for AFB smear therefore Anti Koch's treatment started with four First line dugs. Histopathological examination of the pus and resected material were inconclusive GENE $\mathrm{X}$-PERT was negative. As AKT started we continued it. Patient gradually improved symptoms disappear and on follow up completely cured so AKT stopped after 9 months.

\section{Discussion}

The occurrence of tuberculosis of the middle ear has dramatically decreased, from about $1.3 \%-18.6 \%$ in the beginning of the last century, down to $0.04 \%$ of all cases of chronic suppurative otitis media according to a recent study report [1]. This could be due to better hygiene, improved laboratory facilities, Bacille Calmette-Guerin (BCG) vaccination and a variety of specific drugs [6]. Tuberculosis of the middle ear and mastoid occur as a result of hematogenous or lymphatic spread or by extension to the middle ear cleft through the eustachian tube $[1,3,5,7]$. Tuberculous mastoiditis has a classical presentation. It should be considered in patients with chronic middle ear infection unresponsive to routine antibiotic therapy with painless ear discharge, hearing loss disproportionate to the extent of disease and multiple perforations, in some cases central or total perforation. Facial nerve palsy is rare [8]. Facial nerve palsy associated with tuberculous mastoiditis is seen in approximately $16 \%$ of adult cases and $35 \%$ of paediatric cases [9]. Other findings associated with tuberculosis of the mastoid include the presence of caseous material and granulation tissue seen on otoscopy, which may extend into the mastoid and may sometimes be confused with a cholesteatoma $[6,10]$. Perforation of the tympanic membrane is one of the common features (64-77\%) and may be single or multiple [10]. Involvement of the mastoid is frequently shown on the X-ray and on CT as poor pneumatization or clouding due to granulation tissue or complete bone destruction $[9,10]$. Diagnosing tubercular otitis media requires a high index of suspicion even in the absence of pulmonary tuberculosis. Demonstration of (Acid Fast Bacilli) AFB in the ear discharge is difficult. The positivity for AFB in ear discharge varies from 5 to $35 \%$ and on repeated examinations it improves to $50 \%$ [11], diagnosis of extra pulmonary tuberculosis is essentially clinical $[4,5]$ and antitubercular therapy can be started on clinical or histopathological suspicion [12] as done in our case. It's mandatory to start the treatment early to avoid serious complication. The role of surgery is limited and indications for surgical intervention include cases unresponsive to medical therapy and extensive disease with bone sequestrae.

This case highlights a number of potential issues leading to the delay in diagnosis, which is a hallmark of tuberculous mastoiditis. Although rare, the classical description of tuberculous otitis, reported in literature, includes painless otorrhea, facial palsy and multiple perforations $[3,5,6]$. Contrary to this classical description, in our case there was no evidence of otorrhea or facial paralysis, and any perforation was observed by otoscopy. However, the patient was suffering from hearing loss. In literature, it might range from moderate conductive hearingloss to cophosis [1]. One or multiple perforations in the tympanic membrane, with polypoid middle ear mucosa are typical of the disease. Our patient had an inflammatory bulging of the tympanic membrane, without perforations. At an advanced stage of the disease, the eardrum or the atrium can present a granulomatous or necrotic aspect, and bony sequestrum occurs frequently [4]. The occurrence of pre-auricular or cervical lymph nodes is not uncommon, and development of retroauricular fistula is usual [1-4]. Our patient had a mastoiditis associated with left retro-auricular fistula and cervical lymph nodes. Tuberculous mastoiditis may cause serious complications. When the infection spreads from the ear canal to the nearby tissues such as the skull base or the tegmen tympani, tuberculous mastoiditis could lead to skull base osteomyelitis or tuberculous meningitis [1]. According to the study reported by Rho, et al. [7], CT scan of the temporal bones showed, in most cases, soft tissue attenuation in the entire middle ear cavity, preservation of the mastoid air cells without sclerotic change, and soft tissue extension to the external auditory canal (EAC) or mucosal thickening of the bony EAC. In our case, the CT scan showed total destruction of mastoid air cells with multiple bony sequestrae, and bone cavities filled in the left middle ear resumes some CT scan results reported in literature.

\section{Conclusion}

Tuberculous mastoiditis is very rare. Its diagnosis requires a high index of suspicion. Untreated tuberculous mastoiditis can result in permanent, severe sequel, such as facial paralysis, hearing impairment, and intracranial dissemination of infection. Early suspicion and timely diagnosis are of great importance in resolution of disease and prevention of such serious complications [11]. We have reported a rare presentation of tubercular mastoiditis treated successfully with antitubercular treatment.

\section{Consent}

Obtained from the patient.

\section{Competing interests}

None to declare.

\section{Acknowledgement}

We are thankful to Sister Lucin CEO, Dr. Armida Fernandees Ex Medical director, Dr. Neeraj Uttamani Medical Director, Dr.(Sister) Bina Asst. CEO, Dr. Melvin Methias Medical Suptd and all the staff who have contributed in preparing this manuscript.

\section{References}

1. Awan MS, Salahuddin I (2002) Tuberculous otitis media: two case reports and literature review. Ear Nose Throat J 81: 792-794. [Crossref]

2. Siqueira-Batista R, Palheta-Neto FX, Gomes AP, Pezzin-Palheta AC (2002) Tuberculosis-related middle ear otitis: a rare occurrence. Rev Soc Bras Med Trop 35: 267-268. [Crossref]

3. Grewal DS, Baser B, Shahani RN, Khanna S (1991) Tuberculous otitis media presenting as complications: report of 18 cases. Auris Nasus Larynx 18: 199-208. [Crossref]

4. Lalit Kant (2004) Editorial Extra-Pulmonary Tuberculosis :Coming out of the Shadows Indian J Tuberc 51: 189-190. 
Vyas PK (2020) Case report: Tuberculous mastoiditis an uncommon presentation of extrapulmonary tuberculosis in a young immunocompetant adult male

5. Ravi kumar A, Senthil K, Prasanna Kumar S, Gopinath, Gaurav Bambha (2007) Primary tubercular mastoiditis-a rare presentation. Sri Ramachandra J Med 4: 129-131.

6. Skolnik PR, Nadol JB Jr, Baker AS (1986) Tuberculosis of the middle ear: review of the literature with an instructive case report. Rev Infect Dis 8: 403-410. [Crossref]

7. Karkera GV, Shah DD (2006) Silent mastoiditis-tuberculous aetiology presenting as facial nerve palsy. Indian J Otolaryngol Head Neck Surg 58: 108-110. [Crossref]

8. Kim CW, Jin JW, Rho YS (2007) Tuberculous otitis media developing as a complication of tympanostomy tube insertion. Eur Arch Otorhinolaryngol 264: 227-230. [Crossref]
9. Hadfield PJ, Shah BK, Glover GW (1995) Facial palsy due to tuberculosis: the value of CT. J Laryngol Otol 109: 1010-1012. [Crossref]

10. Lee PY, Drysdale AJ (1993) Tuberculous otitis media: a difficult diagnosis. J Laryngol Otol 107: 339-341. [Crossref]

11. Manju M, Agarwal DS, Singh NP, Gadre DJ (1995) Tuberculosis of the middle ear-a case report. Ind J Tub 42: 55-56.

12. James R, Vevaina, Roger C. Bone, E (Edwin) Kassoff, Legal Aspects of Medicine.

Copyright: $(02020$ Vyas PK. This is an open-access article distributed under the terms of the Creative Commons Attribution License, which permits unrestricted use, distribution, and reproduction in any medium, provided the original author and source are credited. 